\section{Resolution, Flexibility, and Quality offered by Dual Robinson \\ Backscattered Electron Detectors}

Paula A. Cecere, Topcon Technologies, Inc

Over the years, efficiency of backscattered electron detectors has improved. Robinson has developed a unique system whereby two BSE detectors are used, offering an array of benefits to the SEM operator. The system consists of two detectors with scintillators of dedicated geometrical shape, providing a collection area greater than $2000 \mathrm{~mm}^{2}$. They are situated against the axis of the electron beam, with the right and left signals combining to form the overall image signal - one that is 1.5 times greater than that of a single detector. The omnidirectional image illumination, similar to that attained thorough a traditional SE detector, prevents shadowing due to the $180^{\circ}$ collection angle.

Meanwhile, the creation of low vacuum (environmental, WET) SEMs has necessitated the use of BSE detectors - as secondary electrons cannot survive the near-atmosphere environment. This technology provides microscopists with the means to look at their moist, oily, and/or non-conductive samples in their natural state. While operating in WET SEM mode, the dual detector system improves the resolving power and image quality of traditionally non-SEM-friendly samples.

Dual Robinson Backscattered Electron Detectors have been developed exclusively for Topcon scanning electron microscopes. This dual system provides, in itself, convenience and flexibility. They are as follows:

1. Sharp Stereo Imaging - providing an image similar to that obtained through a secondary electron detector.

2. Omnidirectional Image IIIumination - through $180^{\circ}$, eliminating shadows created by either side of the specimen.
3. Signal intensity - 1.5 times that of a single detector

4. Collection Area - over $2,000 \mathrm{~mm}^{2}$ by scintillators.

5. TV Scan Observation - allowing the operator to quickly scan the visual field, focus the high quality image, and adjust all SEM parameters as needed.

6. Variety of Image Displays - Atomic number contrast imaging, Topographic contrast imaging, Left detector image, and Right detector image.

7. EDS Analysis - The operator can simultaneously observe and analyze the specimen in WET-SEM mode with the sole use of the right detector.

8. Highly Tilted Observation of Sample - Here, the left detector is utilized solely, allowing high resolution observation of a tilted sample.

9. Specimen Observation under Heated Conditions - Samples can be viewed under heated conditions, which is impossible using any semiconductor detector.

Controls on the front panel of the SEM allow the operator to switch quickly and easily between modes, whether it be Topographic Contrast, Left Image Display, etc. To operate in WET SEM, simply depress a button and the fully automatic pneumatic valve will isolate the gun column from the sample chamber. The sample chamber will then pump down to, and maintain, the pressure level set by the SEM operator, between 0.01 and 2.0 Torr.

Dual Robinson Backscattered Electron detectors can be utilized in both high and low vacuum modes - as the core of Topcon's WET-3D system provides unsurpassable benefits. In addition to eliminating time-consuming and sometimes difficult sample preparation, non-conductive samples can be viewed and analyzed with the greatest of ease.

In comparison to a single or solid-state detector, the dual detector system offers greater versatility and enhanced specimen information for the microscopist.

1. Robinson, V.N.E., "Discriminating between Atomic Number and Topographic Contrast. ." J. Electron Micros. Vol. 38 No. 5, 1989

2. Tomatsu, J. "Low Vacuum Observation Systern Wet-10", Topcon Corporation.

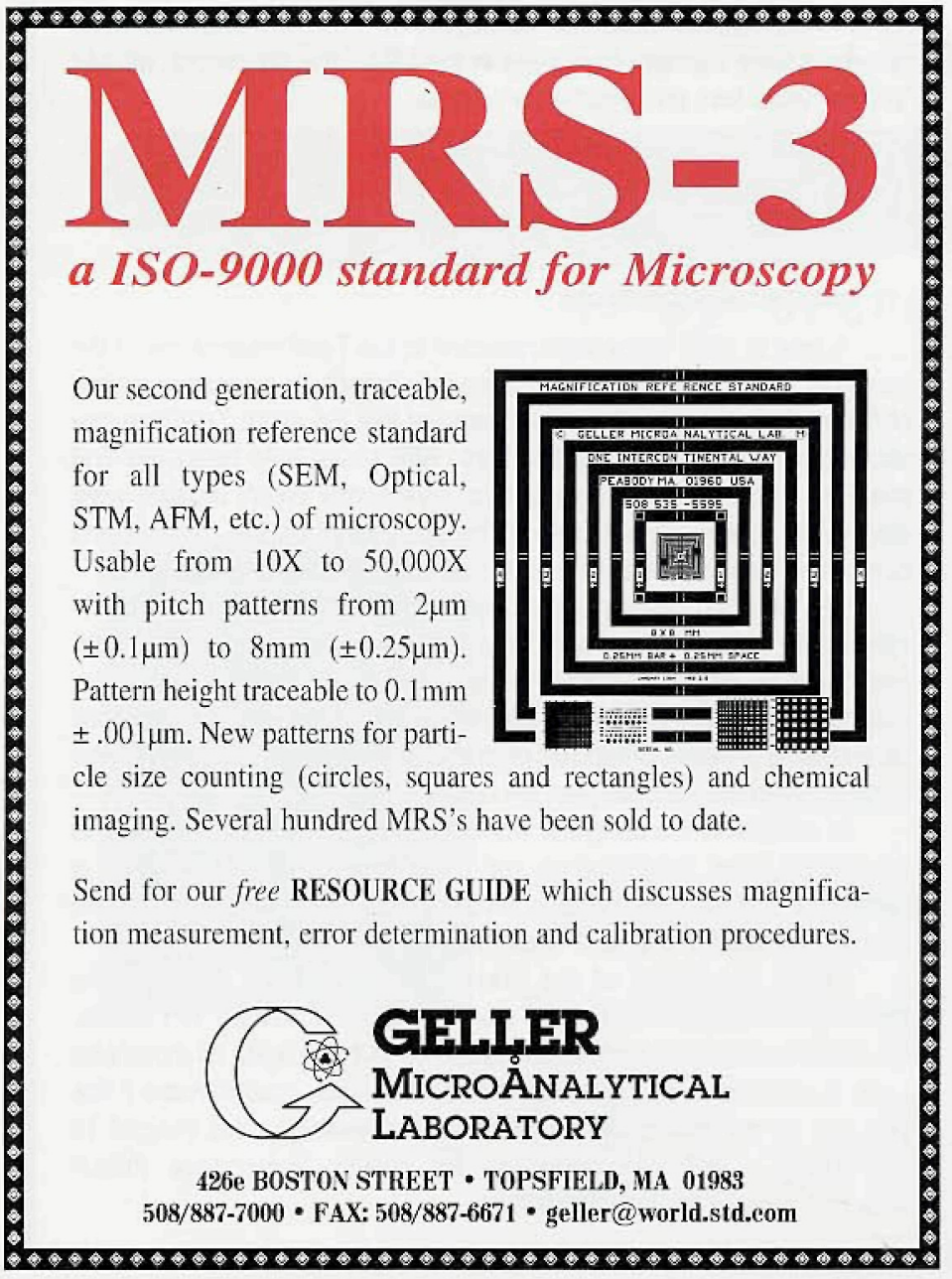

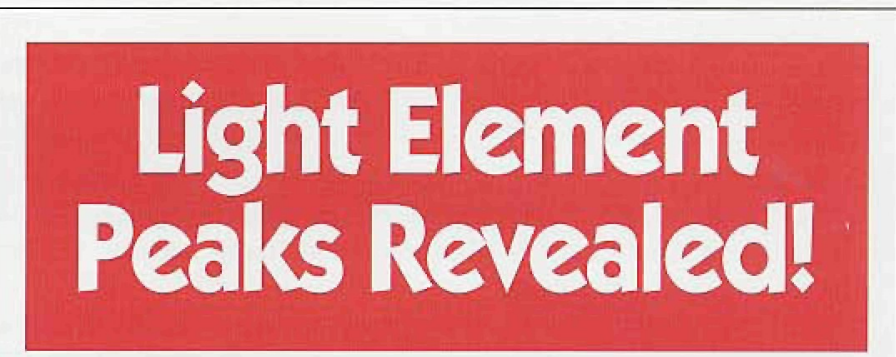

\section{Oil Film on EDX Windows Removed:}

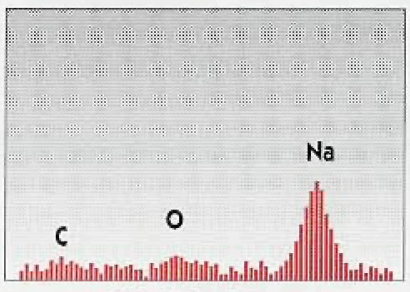

Oily Window

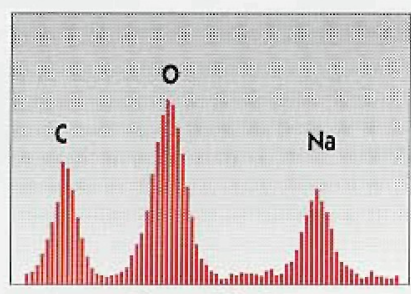

Clean Window
Oil build-up on EDX detector windows can ruin sensitivity for light element X-rays in SEMs. To stop oil condensation and keep the system clean, smart SEM users rely on the XEI Scientific SEM-CLEAN ${ }^{\top M}$ system.

Result: Consistent light element X-ray results and contaminationfree pictures. The Nitrogen purge of the inexpensive SEM-CLEAN system actively cleans your electron microscope while you're away.

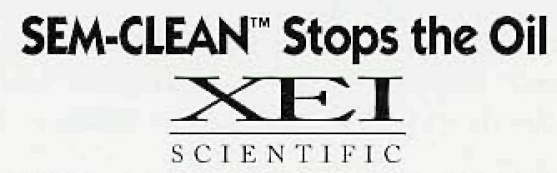

3124 Wessex Way, Redwood City, CA 94061 415-369-0133 - Fax 415-363-1659 


\begin{tabular}{|l|c|c|c|}
\hline \multicolumn{1}{|c|}{ Type of Backscattered Electron Detectors } & $\begin{array}{c}\text { TOPCON WET-3D } \\
\text { Dual Robinson Detectors }\end{array}$ & $\begin{array}{c}\text { Single Robinson } \\
\text { Detector }\end{array}$ & $\begin{array}{c}\text { Solid-State } \\
\text { Detector }\end{array}$ \\
\hline TV scan rate can be used & $\checkmark$ & $\checkmark$ & \\
\hline Heated observation is possible & $\checkmark$ & $\checkmark$ & $\checkmark$ \\
\hline Atomic Number Contrast is obtained easily & $\checkmark$ & & $\checkmark$ \\
\hline Topographic Contrast is obtained easily & $\checkmark$ & & \\
\hline $\begin{array}{l}\text { Image is obtained without shadowing, under } 180^{\circ} \\
\text { illumination }\end{array}$ & $\checkmark$ & $\checkmark$ & \\
\hline $\begin{array}{l}\text { A clear and vivid image can be obtained } \\
\text { simultaneously with EDS Analysis }\end{array}$ & $\checkmark$ & $\checkmark$ & \\
\hline A sample can be clearly observed while highly tilted & $\checkmark$ & & \\
\hline
\end{tabular}

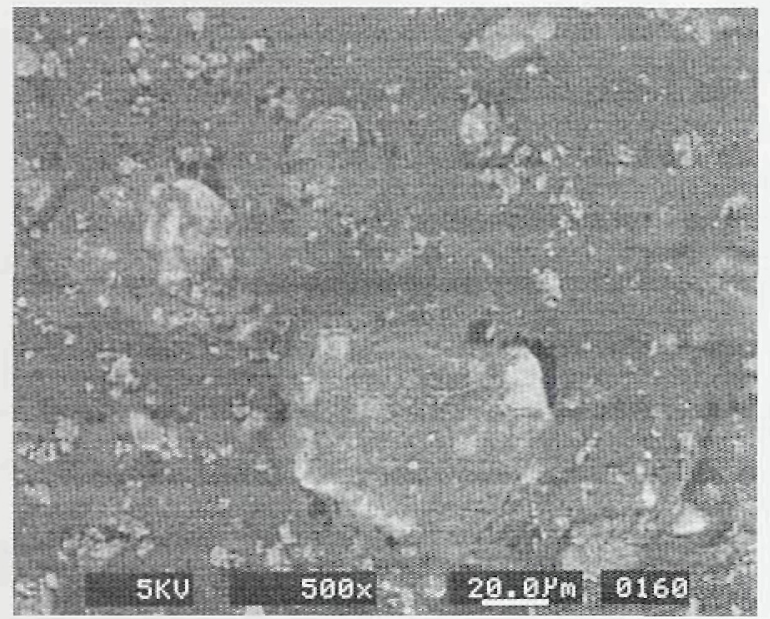

Image 1 -In this picture, atomic number contrast is clearly observed, with an amount of topography visible as well. This is typical of an image obtained using the SET-3D systems in standard mode

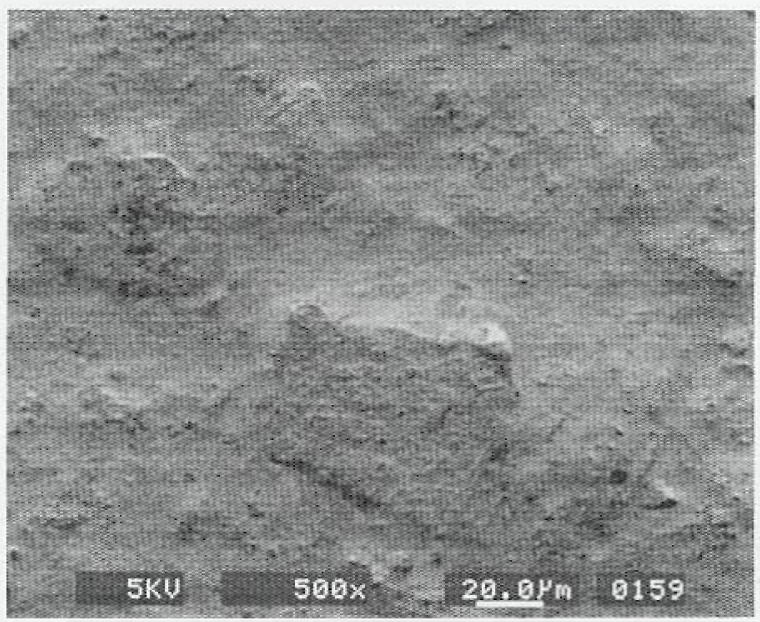

Image 2 - In this picture, the same region was observed with topography as the main component of contrast. The WET-3D system includes a panel button that automatically switches to topographic mode, eliminating the need to mechanically retract the detectors to obtain information on surface details.

\section{STOP HASSLNG WHH
MULTIPLE SERVICE CONIRAGTS}

START by putting all of your instruments under one contract with MAS

(regardless of make or model). Our expert EM SERVICE GROUP has the knowledge and skill to keep your instrumentation working at its best.

\begin{tabular}{|cc|}
\hline \multicolumn{2}{|c|}{ TEM'S/ } \\
\hline HITAM'S \\
JEOL & TOPCON \\
AMRAY & ZEISS \\
CAMBRIDGE & PHILIPS \\
We service all brands under one contract.
\end{tabular}

PREP EQUIPMENT

ULTRAMICROTOMES DUPONT \& RMC VACUUM COATERS SPUTTER COATERS MECHANICAL PUMPS TURBO PUMPS PLASMA ASHERS

\section{WE SERVICE COMPUTER CONTROLLERS} BEAM BLANKERS WATER CHILLERS
Contracts and On-Demand

Emergency Service at

Reasonable Rates from

Factory Trained Specialists.

\section{1-800-421-8451}

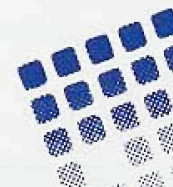

MATERIAIS ANAIYTICAL SERVICES

3597 Parkway Lane • Suite 250 • Norcross, Georgia 30092 • 770-448-3200 • FAX 770-368-8256 or 616 Hutton Street • Suite 101 - Raleigh, North Carolina 27606 • 919-829-7041 • FAX 919-829-5518 ADVANCED ANALYTICAL PRODUCTS AND SERVICES 


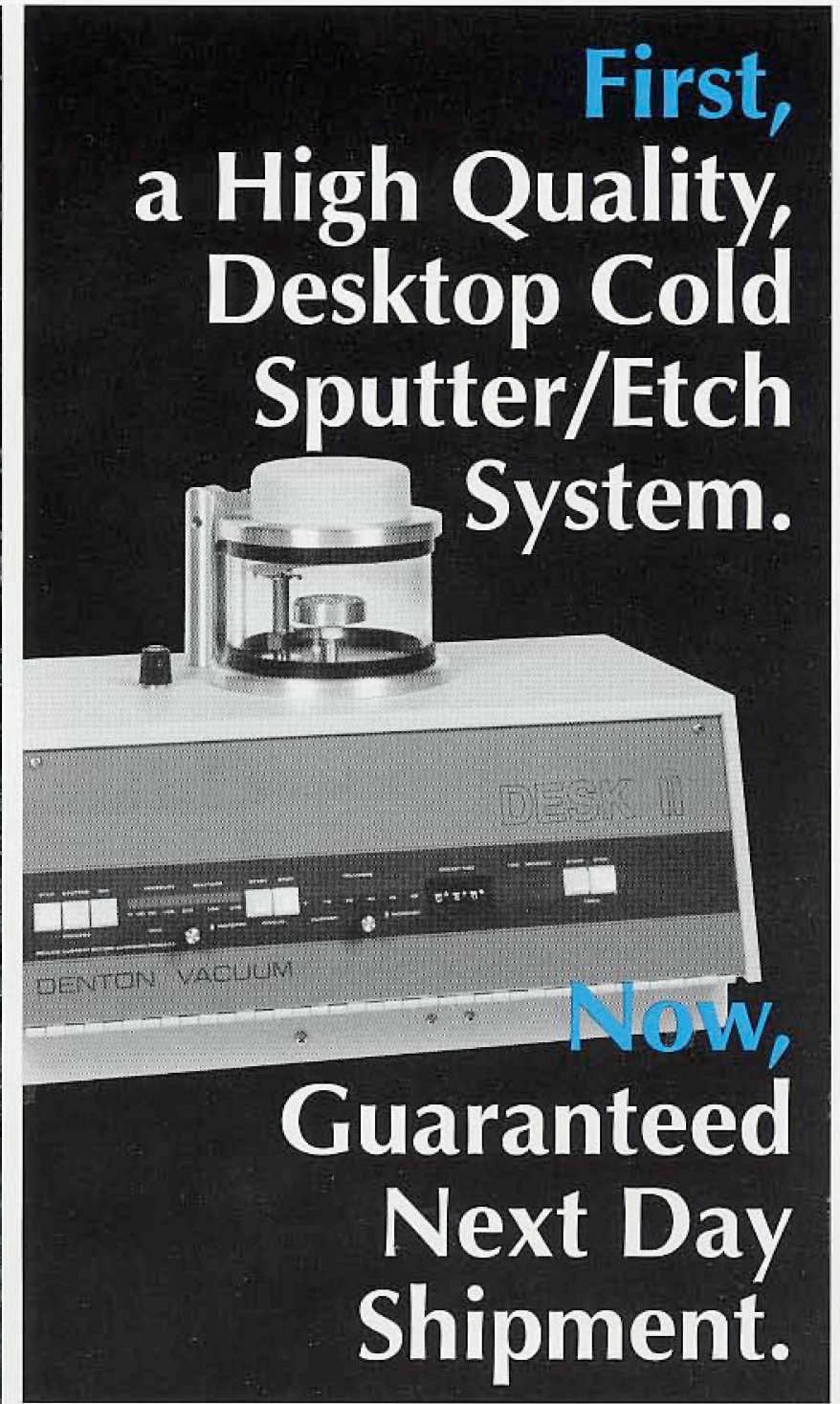

Order Denton's standard Desk II System by noon today and get guaranteed shipment tomorrow or Denton pays the freight.

The Desk II produces uniform, conductive, fine grain $\mathrm{Au} / \mathrm{AuPd}$ coatings in under three minutes and is the highest quality desktop system available.

\section{The Desk I/ features:}

\section{Automatic or manual operation}

- Easy to read digital vacuum and current gauges

Optional carbon evaporation accessory

For more information, look no further than Denton... where both Quality and Delivery invite comparison.

\section{North Church St. ENTON Moorestown, NJ 08057 USA T7ACUUMT Tel: (609) 439-9100 INC. \\ FAX: (609) 439-9111 \\ $\star$ MMDE LI AWBRIOA}

Next day shipment must be requested at time of order.

Decades of Experience In EM Specimen Prep Equipment

\section{coming events}

CCD Imaging Workshops (Photometrics) Tucson, AZ. Lisa Soroka: (520)889-9933, Fax: (520)295-0299.

\section{Oct $3 / 4$ ' 96}

$\checkmark$ First Wed. of Each Month in '96: New Strategies \& Tactics in Image Analysis. lowa City, IA. Dr. J.K. Beddow, (319)337-2427, Fax: (319) $337-2474$.

$\checkmark$ Materials TEM Specimen Preparation Workshops. (AMC Group). Scottsdale, AZ. Dr. Farhad Shaapur: (602)949-4203, Fax: (602)473-9421

Nov. 6/8 '96: FlB Cross-sectioning for SEM/TEM

Nov. 13/15 "97: TEM Wedge-Polishing

Nov. 18/20 '96: Basic Ultramicrotomy

Nov. 21/22 "96: Advanced Ultramicrotomy

$\checkmark$ Sept 18/21 '96: Gold Cluster Labeling Workshop. Brookhaven Nat'I Lab, Upton, NY. Jim Hainfeld, (516)344-3372, Fax: (516)344-3407 $\checkmark$ Sept 20/22 '96: Symposium on Integrated Microscopy (Univ. of Wisconsin \& Carnegie-Mellon Univ.) Madsion, Wl: eMail: http:liwww.bocklabs. wisc.edu/imr/imr.html

$\checkmark$ Sept 26/Oct 2 '96: 14th International EM Congress. Cancun, Mexico. Miguel Jose Yacaman: Tel./Fax: 525-570-85-03

$\checkmark$ Sept 26/27 '96: lowa Microscopy Society Fall Meeting. lowa City, IA. Kenneth C. Moore: (319)335-8142, Fax: (319)335-9049

$\checkmark$ Sept 30/Oct 4 '96: OlM Academy: Course in Orientation Imaging Microscopy. (TSL), Provo, UT. Klaus Behnert: (801)3448990, Fax: (801)344-8997

$\checkmark$ Oct 1/4 '96: Ultramicrotomy for Materials Science Applications Workshop. (RMC \& AZ Materials Lab). Tucson, AZ. Dr. Bob Chiovetti: (520)889-7900, Fax: (520)741-2200.

$\checkmark$ Oct $7 / 11$ '96: Scanning Electron Microscopy and X-Ray Microanalysis for the Materials Scientists. (SUNY). New Palt, NY. Dr. A.V. Patsis: (914)255-0757, Fax: (914)255-0978.

$\checkmark$ Oct 13/16 '96: 5th Brazilian Conference on Microscopy of Materials - MICROMAT 96: Rio de Janeiro. Prof. W.A. Mannheimer, +5521 280-7443, Fax: +5521 290-6626, wamann@metalmat ufrj.br

$\checkmark$ Oct 14/18 '96: 43rd American Vacuum Society National Symposium. Philadelphia, PA. (212)248-0200, Fax: (212)248-0245

$\checkmark$ Oct. 23/30 '96: Optical Microscopy and Imaging in the Biomedical Sciences. Marine Biological Lab., Woods Hole, MA. Carol Harnel: (508)289-7401.

$\checkmark$ Nov. 3/6 '96: Basics of Cryo-Electron Microscopy. (Purdue Univ.) West Lafayette, IN. Susan Umberger: (317)494-7217.

$\checkmark$ Nov. 13 '96: 24th Scottish Microscopy Group Symposium. Aberdeen, Scotland. Kevin MacKenzie: 01224-272847, Fax: 01224272396 .

$\checkmark$ Dec. 2/6 96: Symposium on Materials Issues in Art and Archaeology V. (Smithsonian Inst.) Boston, MA. Pamela Vandiver: (301)238-3700, Fax: (301)238-3709.

$\checkmark$ Dec. 4/6 '96: 26th Annual Conference of the Microscopy Society of Southern Africa. Durban, South Africa Dr. Fiona Graham, +27-31-260-2174, Fax: +27-31-261-6550.

$\checkmark$ Dec. 12/13 '97: Joint Meeting of the Belgian and Dutch Societies for Microscopy. Gent. Nick Schryers: http./I www.ruca.ua.ac.be/'BVM_SBM/progr_net.htm|

$\checkmark$ February 8/14 '97: Photonics West '97. (SPIE). San Jose, CA. Marilyn Gorsuch: (360)676-3290, Fax: (360)647-1445 


\section{ElectroScan's Field Emission ESEM $^{\circledR}$}
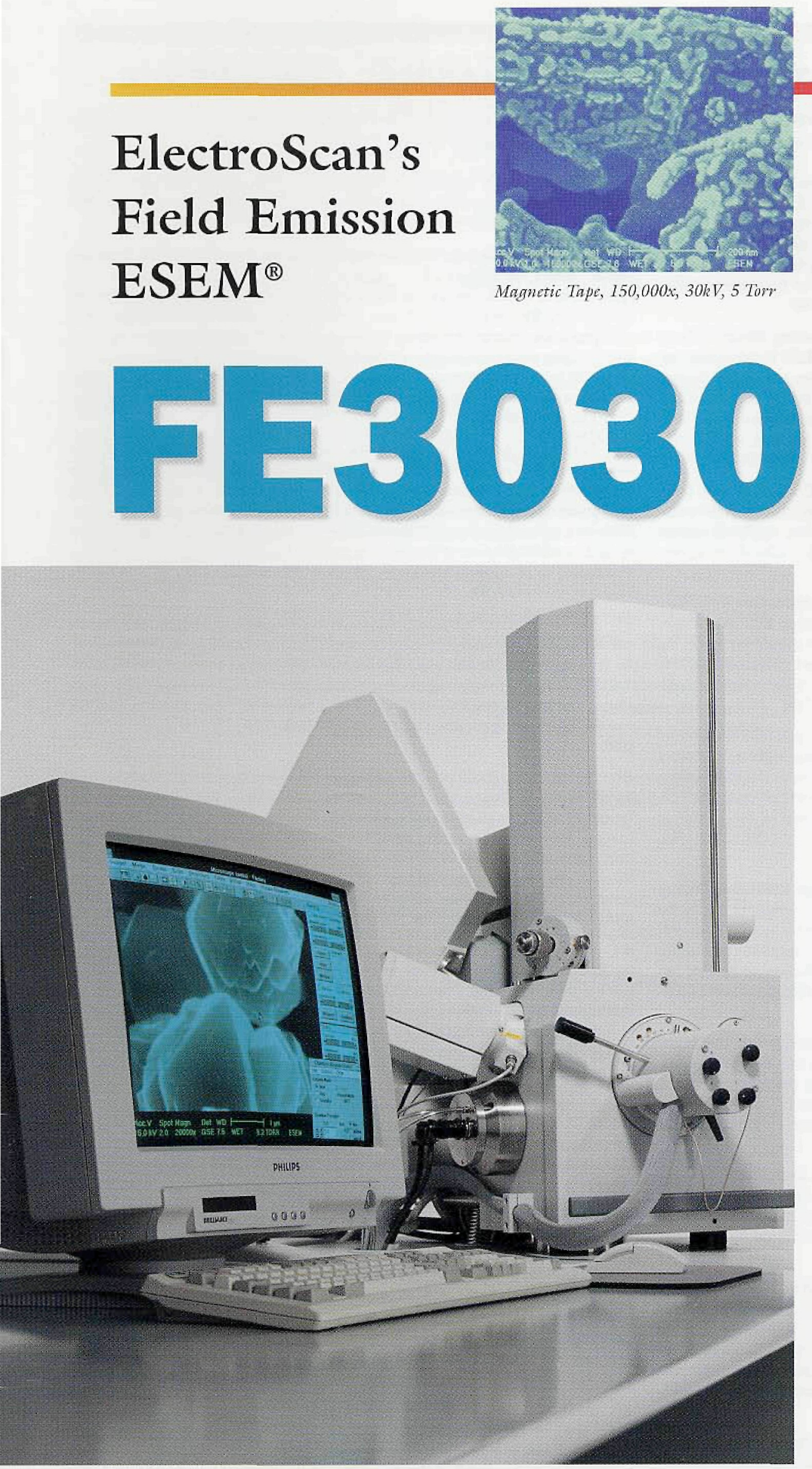

ElectroScan Corporation's ESEM FE3030, an ultra high resolution field emission gun Environmental Scanning Electron Microscope, designed, developed and manufactured jointly with Philips Electron Optics.

ElectroScan, ESEM, and Secing Things You've Never Seen Before are registered trademarks of ElectroSean

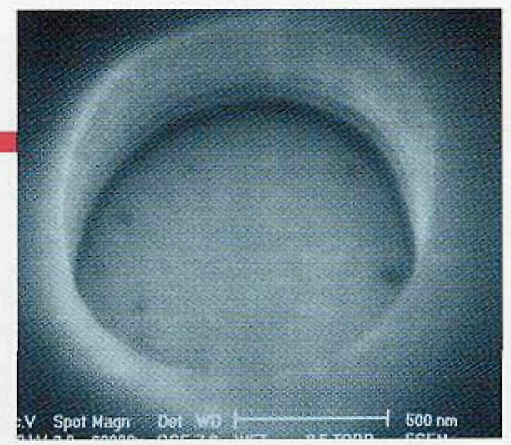

Hole in resist, $60000 x, 5 k \mathrm{~V}, 3.5 \mathrm{Tor} r$

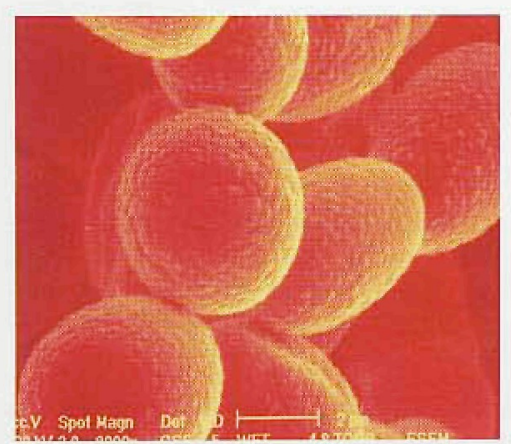

Penicillinum notatum mold, $8000 x$, $5 k V, 5 \mathrm{Tor}$

Environmental Scanning Electron Microscope

$+2 n$ m secondary electron resolution at 7 Torr at $30 \mathrm{kV}$

- High stability Schottky Field Emission gun

+ High beam current capability for $x$-ray on uncoated samples

- High resolution imaging of wet samples with no dehydration

$\uparrow 45^{\circ}$ Conical objective lens

$\downarrow$ Motorized eucentric stage

+ Microscope control from Windows $^{\text {ID }}$

\section{ElectroScan ${ }^{\oplus}$ Corporation}

Seeing Things You've Never Seen Before ${ }^{z}$

66 Concord Street

Wilmington, MA 01887

Tel (508)988-0055

Fax (508) 988-0062

Visit us on the World Wide Webhttp://www.electroscan.com 
As is our custom, and for the hopeful interest of readers who were unable to attend the recent MSA/MAS/MSC Conference in Minneapolis, we have attempted to summarize as follows what was new and/or interesting.

- 4pi Analysis announced the $\mathrm{PCl}$-compatible Spectral Engine II product packages. This state-of-the-art PCI version of the Spectral Engine II allows the acquisition of $x$-ray spectra from EDS pulse processors and high resolution digital images from SEMs and TEMs. EDS/WDS spectral image mapping is also provided. Providing direct acquisition to many affordable commercial software packages, 4 pi's software plug-ins/drivers are compatible with Macintosh and PowerlMac computers. A NuBus version of the hardware is also available. 4PI ANALYSIS, INC.: (919)489-1757, Fax: (919)489-1487

- AMRAY displayed its popular line of $\mathbf{3 0 0 0}$ Series Scanning Electron Microscopes. The 3000 Series product line includes the Model 3200 ECO Variable Pressure SEM that allows uncompromised examinations of unfriendly samples, and the Low Energy Advanced Performance Model 3600 LEAP SEM. The Model 3600 LEAP boasts of a high resolution specification of $4 \mathrm{~nm}$ at $1 \mathrm{kV}$, and $1.5 \mathrm{~nm}$ at $15 \mathrm{kV}$. Other features include a 2048 $\times 2048$ frame buffer, embedded computer control of all SEM functions, and patented Schottky Field Emission. AMRAY INC:: (800)225-1462, Fax: (617)275-0740.

- Barry Scientific displayed the Kimball Physics ES-423E Extended Life LaB6 Cathodes which combines easy operation with exceptional thermal, chemical, mechanical and electrical stability. Also on display were Tungsten filaments, sputter \& carbon coaters and other EM accessories. BARRY SCIENTIFIC, INC: (508)347-9855, Fax: (508)347-8280.

- CamScan introduced their new MaXim scanning electron microscope in the USA The CamScan MaXim is a fully computer-controlled, software-driven SEM with a userfriendly OpenWindows-based graphical user interface. Users can operate the MaXim via mouse/keyboard input, or through a digital manual control knobset. The new MaXim is available as a siand-alone SEM, or as a fully integrated SEMJEDX system utilizing a single host computer. CAMSCAN USA: (412)772-7433, Fax: (412)772-7434.

- The Clemex IMPAK image analysis system can display a spectacular resolution of up to $1600 \times 1200$ pixels. It includes the powerful Clemex Vision software that offers complex functions that, up to now, were only available with expensive image processing boards. In addition, we demonstrated the new Clemex ISAAC, the first fully integrated EDS analysis package for SEM and TEM using 32 bit technology based on WindowsNT or Windows 95 workstations, CLEMEX TECHNOLOGIES, INC: (514)651-6573, Fax: (514)651-9304

- Cressington Scientific Instruments featured it's 208HR High Resolution Coating System for coating difficult samples for FESEM imaging. The unique design of the Rotary-Planetary-Tilting sample stage moves the sample through a non-repetitive path that optimizes the distribution and coverage of the sputtered material. A magnetron head allows for a wide choice of target materials. The chamber design and effective gas handling can achieve totally "cool" sputtering - eliminating any sample heating. CRESSINGTON SCIEN. TIFIC INSTRUMENTS, INC.: (412)772-0220, Fax: (412)772-0249

- Diatome U.S. had on display their complete range of diamond knives for light and electron microscopy at room and cryo temperatures - for materials and biological sciences. As well, their unique diamond trimming tools and anti-static devices were shown. Diatome announced the opening of their unique Web site where the most up to date technical information and sectioning recommendations can be found. DIATOME U.S.: (215)646-1478, Fax: (215)646-8931.

- Digital Instruments announces a powerful extension to its patented TappingMode ${ }^{\text {TM }}$ Atomic Force Microscopy. By mapping the phase of the cantilever oscillation during the scan, phase imaging goes beyond simple topographical mapping by detecting variations in composition, adhesion, friction, viscoelasticity, and other properties. DIGITAL INSTRUMENTS: (805)899-3380, Fax: (805)900-3392

- EDAX introduced their new Phoenix EDS analyzer system for SEM's and TEM's. Phoenix utilizes the WindowsNT operating system and features as standard: a high-end Dual PentiumPRO workstation, digital signal processing, high speed scan generator for fast imaging, true multi-lasking, and a full range of 32-bit application software packages written specifically for WindowsNT. Also featured was the new Sapphire series detector with improved resolution, sensitivity, and peak-10-background specifications. EDAX INTERNA. TIONAL: (201)529-6277, Fax: (201)529-3156

- E. Fjeld Company introduced a series of new products high-lighted with the Beam Current Monitor and Airlock Transfer System. The autoranging, self-contained picoammeter provides quick/easy current monitoring at the sample, with precise, repetitive results for EDS \& WDS analysis. The four inch Transfer System, capable of total integration with a
Field Emission stage, is complete with integrated electronic controls, and eliminates the need to vent Specimen Chambers for sample exchange. E. FJELD CO., INC: (508)6671416, Fax: (508)667-9059.

- Electron Microscopy Sciences displayed for the first time their unique Scanning Digital Camera that captures 36 bit images at up to $2700 \times 3380$ pixel resolution. The system uses a CCD to capture the image field in a single pass and produces images approximately $30 \mathrm{x}$ the resolution of video cameras, which allows for the enlargement of micrographs and micrography images without pixelization. Their Website was introduced which includes many technical tips, and an electronic version of News \& Views 4 , and much more. Old faithful items such as their unique Laboratory Microwave Oven and Oscillating Tissue Slicer were displayed. ELECTRON MICROSCOPY SCIENCES: (215)6461566, Fax: (215)646-8931

- Featured on the stand at MSA was the new, state-of-the-art Polaron range of SEM sample preparation equipment, including the $\mathrm{SC} 7640$ automatic, high resolution sputter coater with its unique annular target and terminating film thickness monitor, the LT7400 SEM cryo-preparation system, and the CPD7501 automatic critical point dryer. For TEM, we previewed our unique Autoprocessor for 1-hour automatic processing of TEM film and the $\mathrm{H} 2800$ Microwave Processor for fast, reproducible fixation, polymerization and immunostaining of biological specimens. ENERGY BEAM SCIENCES, INC. (413)7869322, Fax: (413)789-2786

- ETP-USA received outstanding acclaim on its presentation of the New High Performance Robinson Detector. The Ultra High Resolution BSF images on display, as well as the SEM showing of this new Series 5 detector attested to its superior performance. With over $60 \%$ increase in signal capture and a redesigned scintillator shape allowing $5 \mathrm{~mm}$ working distance, the new Robinson Series 5 is unmatched. ETP-USA also announced its new INTERNET address: www etp-usa.com ETP USA: (510)449-8534, Fax: (510)4498996

- Evex Analytical introduced their next generation of EDS products. Featured was the affordable, PC based VIDX EDS microanalysis system, VIDX Universal Pulse Processor, VIDX Liquid Nitrogen-Free Room Temperature Detector, VIDX Scan active imaging and elemental mapping system. $V / D X C C D$ is an affordable high quality $C C D$ for optical microscopy. Both VIDX Scan and VIDX CCD can be bundled with Image Pro and Materials Pro. Evex Service Engineers will also repair and upgrade Evex, Kevex, Link, Noran, PGT, and Tracor $x$-ray analyzers, and detectors of other manufacturers. EVEX ANALYTICAL: (90B)B74-3800, Fax: (908)874-4647

- FEl Company's Components Group profiled their high resolution, long life Schotthy Emission cathodes which are used by majority of the SEM, TEM and CD-SEM manufacturers in the world. The Components Group announced their new, dedicated facility with expanded space for manufacturing, research and development, and administration. FEl's Systems Group profiled their series of FIB (Focused Ion Beam) Workstations for applications including TEM sample preparation, IC failure analysis, and FIB nanosurgery on biological specimens. FEI COMPANY: (503)640-7500, Fax: (503)640-7509.

- Addressing a long standing need, the new SAMx program, TN 2 WIN, provides a solution for the nagging problem of what to do with all the data which resides in TN $5 \times x \times$ and Series II systems. TN 2 WIN converts spectra and images from both hard and floppy disk environments to WINDOWS format quickly, easily and economically. All that's required is a PC running with WINDOWS 95 and equipped with a bi-directional parallel port. HESSLER TECHNICAL SERVICES: Tel.JFax: (203)358-0266

6. Hitachi Scientific Instruments announced their new H-7500 PC-TEM. The microscope control is through a Windows interface and the user can easily obtain exceptionally high contrast with a large field of view even at low magnification - such as $700 x$. This new $120 \mathrm{kV}$ TEM represents the state-of-the-art in the biological and light materials category. Hitachi also exhibited capabilities of live, remote control of a HP-2000 FE-TEM located in Oak Ridge, TN from the convention center. Hitachi now offers this new technology to the EM community for both TEM and SEM. HITACHI SCIENTIFIC INSTRUMENTS: (415)9691100, Fax: (415) $961-0368$

- JEOL introduced its new JSM-6340F Scanning Electron Microscope. The JSM$6340 \mathrm{~F}$ is a "near-lens, through-the-lens-detector" type of instrument incorporating JEOL's newly designed computer based automation system. This Intuitive User Interface consists of a well though out, unobirusive control panel highlighted by a bright, crisp image on a $1280 \times 1024$ color monitor. The system uses both a mouse and a small digi-knob 
control panel to make the JSM-6340F the most user friendly FE-SEM ever. This network-ready digital SEM features the ultimate low $\mathrm{kV}$ resolution $(2.5 \mathrm{~nm}$ at $1 \mathrm{kV}$ ) and an excellent analytical geometry. JEOL USA, INC.: ((505)535-5900, Fax: (505)536-2205

- Kevex introduced the Quantifier detector series, which provides high throughput while maintaining resolution, now achieving $129 \mathrm{eV}$ resolution at Mn. Kevex has also introduced our Digital Pulse Processsor (DPP), which further enhances the throughput capability of the Quantifer series. The Quantum ATW technology has also been improved which continues to provide Kevex with an $x$-ray transmittance advantage. When combining the improvements in ATW, Crystal \& FET pairing, and the DPP, the Quantifier detector series is now \#1 in performance. KEVEX: (805)295-0019, Fax: (805)295-8714

- Ladd Research Industries has announced an expanded line of apertures/microholes. Apertures as small as five microns are available in a wide variety of metals such as platinum, molybdenum, stainless steel and other materials. Ladd produces single or multiple hole apertures for many applications including electron microscopes, optical, ion beam columns, ion propulsion systems, gas flow and beyond. Ladd will help you design and custom-make your special apertures. LADD RESEARCH INDUSTRIES, INC. ((802)978-6711, Fax: (802)878-8074

* Leica demonsirated instruments for precise microscopic sample preparation. The LEICA UItracut UCT ultramicrotome was featured as well as the LEICA EM FCS cryo-ultramicrotome system, engineered for fast and easy conversion from conventional sectioning at ambient temperature to cryosectioning. Leica's knifemaker which utilizes a balanced break method to produce superior quality iriangular glass knives was also on display. LEICA INC: (708)317-7205, Fax: (708)405-0030.

- Microscopy/Microscopy Education celebrated its fifth anniversary with new brochures and special discounts an its expanded, on-site training programs in microscopy, sample preparation, and image analysis. They have also been chosen as factoryauthorized trainers for Topcon ?SEMs. MME's parent company, Microscopy/Marketing \& Education reached over $20 \%$ of the scientific attendees at this meeting, to gather data profiling the microscopy industry. Both the MSA ' 96 report and a new 5 year retrospective of the microscopy industry are now available. MICROSCOPY/MICROSCOPY EDUCATION: ((413)746-6931, Fax: (413)746-9311

* MOXTEK showed their new dimension standard for scanning probe microscopes (SPM). This submicron feature size standard can be used to calibrate and linearize the scan to $0.5 \%$, just as their existing standards do for scanning electron microscopes. Software that does the work of scan linearization for SPMs was also introduced MOXTEK, INC.: (801)225-0930, Fax: (801)221-1121

- Nissei Sangyo Canada introduced Version 4 of Quartz PCl, the popular Windows 95 based digital image acquisition and archiving system for microscopists. $\mathrm{PCl}$ works with any SEM or TEM, as well as light microscopes, scanners and digital cameras. Version 4 offers many new features including 12 bit deep gray scale images, simultaneous dualchannel acquisition and real-time frame averaging, adding and mixing. PCl's scientific image database capabilities were demonsirated with networked workstations running Windows 95 and WindowsNT server. NISSEI SANGYO CANADA: (416)675-5860, Fax: (416)675-0061.

* NORAN Instruments presented its latest version of WDS Automation and Analysis software for the VOYAGER ${ }^{\circ}$ Microanalysis system. This software can control up to 5 WDS spectrometers and can acquire EDS and WDS data simultaneously. Also shown were the APeX ${ }^{T M}$ and IbeX ${ }^{T M}$ WDS spectrometers, which are part of the product line recently acquired from Peak Instruments. Featuring full computer control of spectrometer components, the NORAN WDS spectrometers are "the next generation of WDS spectrometers." NORAN INSTRUMENTS: ((608)831-6511, Fax: (608)836-7224

- Oxford Instruments introduced Link GSR, gunshot residue search and classification package; Link Opalmap, an automated crystal orientation mapping tool; MiniCL, a low cost cathodoluminescence system; and the XGT-2000W X-ray microscope. Link Opalmap, a simple and easy to use technique, provides automated crystal orientation maps by $X$-rav diffraction in a standard SEM. The XGT-2000W microscope combines the analytical capability of EDS and the penetrating ability of $X$-rays to give simultaneous analysis of a sample's elemental composition and internal structure. OXFORD INSTRU. MENTS: (508)369-9933, Fax: (508)369-8287.

- Philips launched the innovative CM120 BioFilter, a Transmission Electron Microscope that provides Energy Filtered Imaging for the life sciences based on the recently introduced CM120 BioTWIN. The instrument integrates Energy Filter technology and provides excellent image contrast and high collection angle EDX performance. Also demonstrated was the XL30 FEG, a field emission Scanning Electron Microscope that features high analytical performance, fast operation and ease-of-use. PHILIPS ELEC. TRON OPTICS: (201)529-6168, Fax: (201)529-2252

* RJ Lee Instruments Ltd. showcased their new TorrSEM Variable Pressure
System. This extremely affordable package includes the easy-to-use PERSONAL SEM ${ }^{\circ}$, a multi-quad backscatter detector for enhanced imaging, a variable pressure operation package, and online diagnostics. A graphical user interface provides complete vacuum image and accessories control. Clicking an icon switches between high vacuum and high pressure operation - no other user adjustments required. The variable pressure capability is also offered as a retrofit to the standard Model PSM-75. RJ LEE INSTRUMENTS LTD: (412)744-0100, Fax: (412)744-0506

- Small World introduced Version 3.1 of Electron Flight Simulator software for Windows. EFS is a Monte Carlo modeling program for SEM and TEM users who do $X$-ray analysis and need to know critical information about where their $X$-ray data is coming from in the sample. Version 3.1 ads absorption correction and Spectrum Simulator (TM) to make EFS an essential tool in any microanalysis lab. Now you can get a picture of the $X$-rays which get out of the sample, and a click on the Spectra button builds a synthetic spectra of your sample, for your detector and window. A breakthrough in EDS analysis. SMALL WORLD : (415)345-8013, Fax: (415)345-8013.

- South Bay Technology introduced the PC-150 Plasma Cleaner which is a unique device developed to allow simultaneous cleaning of a TEM sample and sample stage which minimizes contamination of inarganic specimens analyzed via SEM, TEM, STEM and/or AEM. The technology involves subjecting the specimen and the specimen stage to a reactive plasma gas which efficiently removes a wide range of contaminants from critical surfaces. The system can be used with any side entry or top entry TEM holder or with any sample that will fit inside the large, easily accessible chamber. SOUTH BAY TECHNOLOGY: (714)492-2600, Fax: (714)492-1499.

- SPI Supplies became the first firm in EM offering sample preparation equipment and consumable supplies via the internet. The central focus of the exhibit booth at MSA was a continuous demonstration of the ease of accessibility of the different products on the web site. As Dr. Charles Garber, President, SPI Supplies pointed out to those coming to the booth, he can put up more information about his products electronically than ever would have been possible with a printed calalog. All prices for all products are given and also, all prices are guaranteed current. SPI SUPPLIES: (800)2424-SPI, Fax: (610)436-5755

- Three emerging electron microscopy technologies were featured by Ted Pella, Inc.

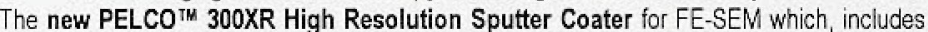
targets of $\mathrm{Cr}$ and $\mathrm{Pt} / \mathrm{Pd}$, was operational during the exhibit. Both blackswhite and color work station printers were demonstrated for direct digital imaging from the microscope. The latest techniques for microwave tissue processing were presented. The PELCOTM Microwave Processor was on display and operating during the show. TED PELLA, INC.: (916)2432200. Fax: (916)243-3761.

- TexSEM Laboratories, Inc. (TSL) introduced Conical Scan Crystallography, a new technique for rapid quantitailve analysis of the crystallographic features of transmission electron microscope (TEM) samples of nanocrystalline thin films and coatings. The technique combines dark field imaging, conical scanning, and TSL's Orientation Imaging Microscopy ${ }^{\text {TH }}\left(O \mathrm{M}^{\mathrm{TH}}\right)$ software. It enables materials scientists to determine grain size, grain boundary morphology, and local texture of thin films and coatings with grain sizes down to approximately 200 Angstroms. Up to 50,000 orientation measurements can be captured in less than one hour of TEM operation. TEXSEM LABORATORIES, INC. (801)344-8990, Fax: (801)344-8997

- Vital Image Technology announced a new 3 and 4 color dye-sublimation network printer. The $3600-40$ printer connects to existing Ethernet (TCP/IP, Ethertalk) networks. This PostScript Level 2 printer has a $260 \mathrm{MB}$ hard disk for data transfer and multi-level password security. VitalScan, a $\$ 9,995.00$ digital imaging system for upgrading any SEM/STEM to a digital microscope was also shown. VitalScan provides the capture of both secondary and backscatter images as well as EDS and WDS maps. VIT has the solution for both network and stand alone imaging applications. VITAL IMAGE TECHNOLOGY: (330)940-3200, Fax: (330)940-3222

- Larger Field-of-View Surface Texture Measurements, now available exclusively from WYKO, is a new and easy method for measuring surface texture of large areas up to several inches. This unique system option, "stitching", makes it possible to automatically measure large surface areas of a sample without sacrificing critical lateral resolution, vertical resolution, range and accuracy. The system automatically takes a series of measurements and seamlessly combines them to create one composite data set analyzing the entire surface. WYKO CORPORATION: (520)741-1044, Fax: (520)294-1799.

- XEI Scientific introduced two new anti-contamination products to compliment the SEM-CLEAN nitrogen purge system. The new "Chill Out" TM cold finger is a refrigerant cooled system that uses a Joule-Thompson cold head instead of LN for cooling. It features vibration free operation and fasi cool down and warm up. If can be used in non-air locked SEMS. Also introduced was a foreline leak system to reduce rotary pump backstreaming as described by Michael Postek, Scanning, Vol 18, 269-284 (June 1996). XEI SCIENTIFIC (415)369-0133, Fax: (415)363-1659. 